\title{
Restored insulin-sensitivity in IRS-1-deficient mice treated by adenovirus-mediated gene therapy
}

\author{
Kohjiro Ueki, ${ }^{1}$ Toshimasa Yamauchi, ${ }^{1}$ Hiroyuki Tamemoto, ${ }^{1}$ Kazuyuki Tobe,${ }^{1}$ \\ Ritsuko Yamamoto-Honda, ${ }^{1}$ Yasushi Kaburagi, ${ }^{1}$ Yasuo Akanuma, ${ }^{2}$ Yoshio Yazaki, ${ }^{1}$ \\ Sininchi Aizawa, ${ }^{3}$ Ryozo Nagai, ${ }^{1}$ and Takashi Kadowaki ${ }^{1}$ \\ ${ }^{1}$ Department of Internal Medicine, Graduate School of Medicine, University of Tokyo, Tokyo, Japan \\ ${ }^{2}$ The Institute for Diabetes Care and Research, Asahi Life Foundation, Tokyo, Japan \\ ${ }^{3}$ Department of Morphogenesis, Institute of Molecular Embryology and Genetics, Kumamoto University School of Medicine, \\ Kumamoto, Japan \\ Address correspondence to: Takashi Kadowaki, Department of Internal Medicine, Graduate School of Medicine, \\ University of Tokyo, 7-3-1 Hongo, Bunkyo-ku, Tokyo 113, Japan. Phone: 81-3-5800-8818; Fax: 81-3-5689-7209; \\ E-mail: Kadowaki-3im@h.u-tokyo.ac.jp. \\ Kohjiro Ueki's present address is: Research Division, Joslin Diabetes Center, Boston, Massachusetts, USA. \\ Received for publication June 22, 1999, and accepted in revised form April 4, 2000.
}

Insulin resistance is commonly observed both in overt diabetes and in individuals prone to, but not yet manifesting, diabetes. Hence the maintenance or restoration of insulin sensitivity may prevent the onset of this disease. We previously showed that homozygous disruption of insulin receptor substrate-1 (IRS1) in mice resulted in insulin resistance but not diabetes. Here, we have explored the mechanism of systemic insulin resistance in these mice and used adenovirus-mediated gene therapy to restore their insulin sensitivity. Mice expressing the IRS-1transgene showed almost normal insulin sensitivity. Expression of an IRS-1 mutant (IRS-1 $\Delta$ p85) lacking the binding site for the p85 subunit of phosphatidylinositol 3-kinase (PI3K) also restored insulin sensitivity, although PI3K is known to play a crucial role in insulin's metabolic responses. Protein kinase B (PKB) activity in liver was decreased in null mice compared with the wild-type and the null mice expressing IRS- 1 or IRS- $1 \Delta \mathrm{p} 85$. In primary hepatocytes isolated from null mice, expression of IRS-1 enhanced both PI3K and PKB activities, but expression of IRS- $1 \Delta \mathrm{p} 85$ enhanced only PKB. These data suggest that PKB in liver plays a pivotal role in systemic glucose homeostasis and that $\mathrm{PKB}$ activation might be sufficient for reducing insulin resistance even without full activation of PI3K.

J. Clin. Invest. 105:1437-1445 (2000).

\section{Introduction}

Insulin resistance is one of the major symptoms of diabetes especially in patients with type 2 diabetes and is usually observed even in a prediabetic state (1). Insulin resistance is thought to be caused by the disturbance of insulin's signaling pathway at various steps (2). We and others previously showed that disruption of insulin receptor substrate-1 (IRS-1), which is the major target of insulin receptor, resulted in insulin resistance and growth retardation, but not diabetes, probably owing to compensatory hyperinsulinemia $(3,4)$. However, we demonstrated that the double knockout mice with disruption of IRS- 1 and $\beta$-cell-specific glucokinase genes developed overt diabetes (5). Bruning et al. also showed that the double heterozygous knockout of IRS-1 and insulin receptor could be diabetic (6). Recently, Withers et al. demonstrated that disruption of IRS-2 resulted in severe diabetes owing to insulin resistance and disturbance of insulin secretion (7). These findings suggest the one possible mechanism developing type 2 diabetes may be failure of compensatory insulin secretion against insulin resistance (8). Therefore, reducing insulin resistance is probably useful in preventing the risk of developing diabetes for prediabetic subjects or in protecting remaining $\beta$-cell function of diabetic patients. Indeed, body weight reduction often improves insulin resistance and blood glucose control.

Many studies suggest that skeletal muscle is the major site for glucose disposal in the human body and also suggest that disturbance of insulin signaling in skeletal muscle plays an important role on developing insulin resistance and diabetes (9). However, liver is the other major target of insulin, and liver dysfunction such as liver cirrhosis often causes postprandial hyperglycemia and insulin resistance. Indeed, many reports claimed that insulin resistance in liver is also important in wholebody insulin resistance (10). Recent studies using transgenic animals have also demonstrated that impairment of insulin signaling in skeletal muscle alone $(11,12)$ or skeletal muscle and adipose tissue (13) is not sufficient for developing diabetes.

To clarify this controversial problem, we attempt to express the wild-type and a mutant IRS- 1 into IRS- 1 knockout mice using adenovirus-mediated gene transfer because it can introduce the gene efficiently in liver by systemic administration (14). Here, we demonstrate 
that restoration of IRS-1 in the IRS-1-deficient mice liver can normalize whole-body insulin sensitivity. We also show the possible mechanism of insulin signaling pathway regulating glucose homeostasis through IRS1 , using primary cultured hepatocytes from IRS-1-deficient mice with adenovirus-mediated IRS-1 expression.

\section{Methods}

Animals. Mice lacking IRS-1 were generated as described elsewhere (3). IRS-1 $1^{+/+}$mice and $I R S-1^{-/-}$mice were used for the following experiments. Mice were bred on normal chow and maintained using standard husbandry procedures. The animal care and procedures were approved by the Animal Care Committee of the University of Tokyo.

Insulin tolerance test. Male mice (12-16 weeks of age) were fed freely and then fasted during the study. They were intraperitoneally challenged with $0.75 \mathrm{mU} / \mathrm{g}$ (body weight) human insulin (Novoslin R; Novo Nordisk, Bagsraerd, Denmark). Blood samples were drawn at the indicated time point from the tail vein (5).

Adenovirus-mediated gene transfer. IRS-1-deficient mice were injected with adenoviruses at a concentration of 3 $\times 10^{8}$ plaque-forming units ( $\mathrm{pfu}$ ) per gram of body weight. Viral suspensions in $200 \mu \mathrm{L}$ of PBS were injected through the tail vein. At 3-5 days after injection, mice were subjected to experiments. In the case of cultured cells, cells were treated with adenovirus at a concentration indicated as moi in each experiment. Infection efficiency was checked by $\beta$-galactosidase assay (15). Briefly, a frozen section of liver of mouse treated with the adenovirus encoding lac $Z$ was fixed with $0.5 \%$ of glutaraldehyde in PBS and was washed extensively with PBS. $\beta$-Galactosidase activity was detected by immersing in 5-bromo-4-chloro-3-indolyl $\beta$-D-galactopyranoside (X-gal) staining solution containing 35 $\mathrm{mM} \mathrm{K}_{4} \mathrm{Fe}(\mathrm{CN})_{6}, 35 \mathrm{mM} \mathrm{K}_{3} \mathrm{Fe}(\mathrm{CN})_{6}, 1 \mathrm{mM} \mathrm{MgCl}_{2}$ and 1 $\mathrm{mg} / \mathrm{mL}$ of $\mathrm{X}$ gal for 15 hours at $37^{\circ} \mathrm{C}$.

Generation of adenoviruses. cDNA of rat IRS-1 (IRS-1wt) including the whole coding region was subcloned into $\mathrm{m} 13$ vector. $\mathrm{cDNA}$ encoding a mutant substituted tyrosine residues of eight YXXM motifs of IRS- 1 into phenylalanine (IRS-1 $\Delta$ p85) was generated as described previously (16). cDNA of IRS-1wt and that of IRS$1 \Delta$ p85 were introduced to the expression cosmid cassette by blunt-end ligation into SwaI site, respectively, as described previously (17). The recombinant adenoviruses Adex1CAIRS-1wt and Adex1CAIRS-1 $1 \mathrm{p} 85$ were constructed by homologous recombination between the expression cosmid cassette and parental virus genome as described elsewhere (17). The control adenovirus, Adex1CALacZ, and the cosmid cassette were kindly provided by I. Saito (University of Tokyo).

Hepatocytes culture. Primary hepatocytes were isolated from $I R S-1^{+/+}$or IRS-1-/- male mice according to Ledley et al. (18) with slight modification. The liver was perfused by cannulation in the portal vein with HBSS containing $0.125 \mathrm{mM}$ EGTA to wash out erythrocytes. Then the liver was sequentially perfused with HBSS containing $0.3 \mathrm{mM} \mathrm{CaCl}_{2}, 100 \mathrm{U} / \mathrm{mL}$ of collagenase, and $120 \mu \mathrm{g} / \mathrm{mL}$ of trypsin inhibitor. The liver was removed and filtered through muslin followed by washing with William's E medium. The cells were resuspended in William's E medium supplemented with $10 \%$ FBS and plated onto a 60 -mm type I collagencoated dish at a density of $1 \times 10^{6}$ cells per dish. After 8 hours, the cells were infected with the adenovirus as already described here and were cultured in William's E containing $5 \mathrm{nM}$ dexamethasone for 24 hours.

Antibodies. Rabbit polyclonal anti-IRS-1 antibodies ( $\alpha$ IRS-1-CT) were generated against a peptide corresponding to the sequence of $\mathrm{COOH}$-terminal 14 amino acids of rat IRS-1 (Upstate Biotechnology Inc., Lake Placid, New York, USA). Rabbit polyclonal anti-PKB antibodies $(\alpha \mathrm{PKB}-\mathrm{CT})$ were generated against a peptide corresponding to the sequence of 465-480 human PKB (Upstate Biotechnology Inc.). Mouse monoclonal antiphosphotyrosine antibodies (4G10) were purchased from Upstate Biotechnology Inc. Rabbit polyclonal anti-MAP kinase antibodies $(\alpha C 92)$ were generated against a peptide encoding $\mathrm{COOH}$-terminus 14 amino acids of rat Erk1 as described previously (19).

Immunoprecipitation and pull down using GST fusion protein from the tissue and cell lysates. Mice were anesthetized, and $1 \mu \mathrm{g} / \mathrm{g}$ body weight of insulin was injected through the inferior vena cava. After the indicated period, the liver, soleus muscle, or epididymal fat pad was removed and homogenized with the tissue homogenization buffer containing $25 \mathrm{mM}$ Tris- $\mathrm{HCl}$ ( $\mathrm{pH}$ 7.4), $10 \mathrm{mM}$ $\mathrm{Na}_{3} \mathrm{VO}_{4}, 100 \mathrm{mM} \mathrm{NaF}, 10 \mathrm{mM} \mathrm{Na}_{4} \mathrm{P}_{2} \mathrm{O}_{7}, 10 \mathrm{mM}$ EGTA, $10 \mathrm{mM}$ EDTA, $5 \mu \mathrm{g} / \mathrm{mL}$ leupeptin, $5 \mu \mathrm{g} / \mathrm{mL}$ aprotinin, $1 \mathrm{mM}$ PMSF, and 1\% NP40. The homogenates were subjected to immunoprecipitation with $\alpha$ IRS-1-CT, $4 \mathrm{G} 10, \alpha \mathrm{C} 92$, or $\alpha \mathrm{PKB}-\mathrm{CT}$. In the case of hepatocytes, cells were treated with insulin and lysed with buffer $\mathrm{A}$ containing $25 \mathrm{mM}$ Tris- $\mathrm{HCl}$ (pH 7.4), $2 \mathrm{mM} \mathrm{Na}_{3} \mathrm{VO}_{4}$, $10 \mathrm{mM} \mathrm{NaF}, 10 \mathrm{mM} \mathrm{Na}_{4} \mathrm{P}_{2} \mathrm{O}_{7}, 1 \mathrm{mM}$ EGTA, $1 \mathrm{mM}$ EDTA, $5 \mu \mathrm{g} / \mathrm{mL}$ leupeptin, $5 \mu \mathrm{g} / \mathrm{mL}$ aprotinin, $1 \mathrm{mM}$ PMSF, and 1\% NP40. The lysates were subjected to immunoprecipitation with $\alpha$ IRS-1-CT, 4G10, or $\alpha$ PKB$\mathrm{CT}$. The immunoprecipitates were immobilized on protein A- or G-Sepharose beads followed by extensive washing with the tissue homogenization buffer or buffer A. For pull-down study, the cell lysates were incubated with $1 \mu \mathrm{g}$ of GST-p85PI3K or GST-Grb2 (20) and immobilized on glutathione Sepharose beads followed by extensive washing with buffer A.

Phosphatidylinositol 3-kinase assay. The immunoprecipitates with 4G10 were washed three times with the tissue homogenization buffer or buffer $A$ and twice with phosphatidylinositol 3-kinase (PI3K) reaction buffer (20 mM Tris- $\mathrm{HCl}$ [pH 7.4], $100 \mathrm{mM} \mathrm{NaCl}$, and $0.5 \mathrm{mM}$ EGTA) and were suspended in $50 \mu \mathrm{L}$ of PI3K reaction buffer containing $0.1 \mathrm{mg} / \mathrm{mL}$ of PI (PI: bovine liver; Avanti Polar Lipids, Alabaster, Alabama, USA). The reactions were initiated by adding $5 \mu \mathrm{L}$ of a $\mathrm{MgCl}_{2}$-ATP mixture $\left(200 \mathrm{mM} \mathrm{MgCl}_{2}, 200 \mu \mathrm{M}\right.$ ATP) containing 5 $\mu \mathrm{Ci}$ of $\left[\gamma^{32} \mathrm{P}\right] \mathrm{ATP}$ to the immunoprecipitates and incubated at $25^{\circ} \mathrm{C}$ for 20 minutes. The reactions were ter- 
minated by adding $150 \mu \mathrm{L}$ of chloroform-methanol$11.6 \mathrm{~N} \mathrm{HCl}$ (100:200:2). After adding $120 \mu \mathrm{L}$ of chloroform to each sample, the organic phase was separated by centrifugation, and it was washed twice with methanol-1N HCl (1:1). After evaporation, the pellets were resuspended in $20 \mu \mathrm{L}$ of chloroform, spotted onto a Silicagel 60 plate (Merck KGaA, Darmstadt, Germany), and developed in chloroform-methanol-28\% ammonium hydroxide-water (43:38:5:7). The phosphorylated lipids were visualized by autoradiography.

In vitro kinase assay. The liver removed from the mouse was lysed with the tissue homogenization buffer, or the primary cultured hepatocytes were lysed with buffer $A$ as already described here, and the lysates were subjected to immunoprecipitation with $\alpha$ PKB-CT or $\alpha$ C92, followed by PKB kinase assay or MAP kinase assay (17). Briefly, the immunoprecipitates with $\alpha \mathrm{PKB}-\mathrm{CT}$ or $\alpha \mathrm{C} 92$ were washed and resuspended in $50 \mathrm{mM}$ Tris- $\mathrm{HCl}$ ( $\mathrm{pH} 7.5), 10$ $\mathrm{mM} \mathrm{MgCl}$, and $1 \mathrm{mM}$ dithiothreitol, to which $50 \mu \mathrm{M}$ ATP, $3 \mu \mathrm{Ci}\left[\gamma^{32} \mathrm{P}\right] \mathrm{ATP}$, and $1 \mu \mathrm{g}$ of Crosstide in PKB assay or $5 \mu \mathrm{g}$ of myelin basic protein (MBP) in MAP kinase assay, had been added, respectively. After $20 \mathrm{~min}$ utes at $30^{\circ} \mathrm{C}$, the reaction was stopped and the aliquots were spotted onto squares of P-81 paper, washed, and counted by scintillation counter.

\section{Results}

Expression of the wild-type IRS-1 into IRS-1-deficient mice by adenovirus-mediated gene transfer. To clarify the impact of liver on whole-body insulin resistance, we introduced IRS-1wt into IRS-1-deficient mice liver by adenovirusmediated gene transfer (21). First, we checked the expression levels of the introduced IRS-1 protein in liver, skeletal muscle, and adipose tissue, which are major insulin target tissues. As expected, IRS-1 was detected in liver to the almost same extent as in the wild-type mice under our infection condition, whereas we could not detect IRS-1 expression in either the skeletal muscle or adipose tissue in most animals (Figure 1a). To assess the expression pattern of the introduced protein in liver, we performed $\beta$-galactosidase assay using the frozen section of the liver of the animal treated with adenovirus encoding LacZ. Eighty percent of hepatocytes exhibited strong $\beta$-galactosidase activity with an almost equal intensity, whereas other components of the liver, such as endothelial cells, were much less intensely stained (data not shown). Furthermore, this exogenous IRS-1 in the liver was tyrosine phosphorylated as much as the one in wild-type mice after insulin injection through the inferior vena cava (Figure 1b). Second, to explore the effect of this liver-specific restoration of IRS-1 on the glucose metabolism of IRS-1-deficient mice, we performed an insulin tolerance test (ITT) on the mice treated with the adenoviruses because IRS-1-deficient mice show insulin resistance in ITT but with almost normal glucose tolerance, as do some prediabetic patients or those with syndrome $X(3,4,22)$. Restoration of IRS- 1 in the liver by adenovirus-mediated gene transfer increased insulin sensitivity in ITT to the almost same level as in the wild-type mice, whereas the mice expressing LacZ showed insulin resistance as much as did IRS-1-deficient mice without infection (Figure 1c). These data were comparable to the expression and phosphorylation levels of IRS-1 in the liver of each type of mice. Third, we investigated the major downstream events from IRS-1, MAP kinase (MAPK) activity, and phosphatidylinositol 3-kinase (PI3K) activity. As Figure 1d shows, MAPK activity in the mouse liver-infected IRS$1 w t$ adenovirus tended to increase compared with that in the one infected LacZ adenovirus. On the other hand, expression of IRS-1 significantly increased in insulin-stimulated PI3-K activity in the liver compared with the mice infected with LacZ adenovirus to the same level as in wild-type mice (Figure 1e).

Expression of the mutant IRS-1 lacking $p 85$ binding sites into IRS-1-deficient mice by adenovirus-mediated gene transfer. Previous studies have shown that PI3K is the major mediator of insulin's metabolic signaling including glucose transport and glycogen synthesis $(23,24)$. It has also been shown that IRS- 1 is the main mediator of PI3K-dependent signaling in response to insulin in many cells and tissues. To clarify the role of PI3K on this recovery of insulin sensitivity in this case, we constructed the adenovirus encoding a mutant IRS-1 that lacks all of p85 binding motifs (YXXM) (25) by substitution of $\mathrm{Y} \rightarrow \mathrm{F}$ in the motifs, designated as IRS- $1 \Delta \mathrm{p} 85$. We characterized this mutant by means of transient expression into COS1 cells expressing human insulin receptor. IRS- $1 \Delta \mathrm{p} 85$ was expressed to the same level as IRS- 1 wt at the same virus titer. However, tyrosine-phosphorylation of IRS- $1 \Delta$ p 85 was prominently decreased comparable to the decrease in the possible phosphorylation sites on this protein (Figure $2 a$ ). To confirm that this mutant lost the ability to bind p85PI3K, a pulldown study using GST-p85PI3K fusion protein was performed. IRS- $1 \Delta \mathrm{p} 85$ was unable to be pulled down with GST-p85PI3K fusion protein, whereas IRS-1wt efficiently bound it (Figure $2 \mathrm{~b}$ ). By contrast, IRS- $1 \Delta \mathrm{p} 85$ seemed to keep the ability to bind Grb2 as much as did IRS-1wt (Figure 2b). Moreover, PI3K activity associated with tyrosine-phosphorylated proteins in cells expressing IRS- $1 \Delta$ p 85 was detectable to the almost same extent as the one in cells expressing LacZ, whereas expression of IRS-1wt significantly increased the PI3K activity (Figure $2 \mathrm{c}$ ). Thus, IRS- $1 \Delta \mathrm{p} 85$ was expected to lose the ability to associate PI3K activity but keep the ability to mediate other signals, such as the signal to MAP kinase activation. It is likely that using this mutant enabled us to reveal the roles of PI3K-dependent and independent pathways mediated by IRS-1 in glucose homeostasis. Therefore, we injected the virus encoding IRS- $1 \Delta$ p 85 into IRS-1-deficient mice and investigated expression and tyrosine phosphorylation of this mutant and PI3K activity (Figure 3a). The mutant IRS-1 protein was mostly detected in the liver as much as that of IRS-1wt by Western blot analysis. IRS- $1 \Delta \mathrm{p} 85$ was phosphorylated by insulin stimulation 
but much less than was IRS-1wt. PI3K activity in the liver expressing IRS- $1 \Delta \mathrm{p} 85$ was activated no more than the one in the liver expressing LacZ. Unexpectedly, in spite of reduced PI3K activity, the IRS-1-deficient mice-infected IRS- $1 \Delta \mathrm{p} 85$ adenovirus recovered insulin sensitivity in ITT to the almost same level as did the mice infected with IRS-1wt (Figures 1d and 3b). To clarify the mechanism of this recovery of insulin sensitivity by IRS- 1 wt or IRS- $1 \Delta \mathrm{p} 85$, it is important to iden- tify the signal that is equally activated by both types of IRS-1. To this end, primary cultured hepatocytes seem to be one of the ideal systems because they are equipped with the similar functions and characteristics as those in vivo.

The effect of the wild-type and the mutant IRS-1 expression on insulin signaling in primary cultured bepatocytes from the IRS-1-deficient mice. We introduced IRS-1wt and IRS$1 \Delta \mathrm{p} 85$ into the primary cultured hepatocytes from IRS-
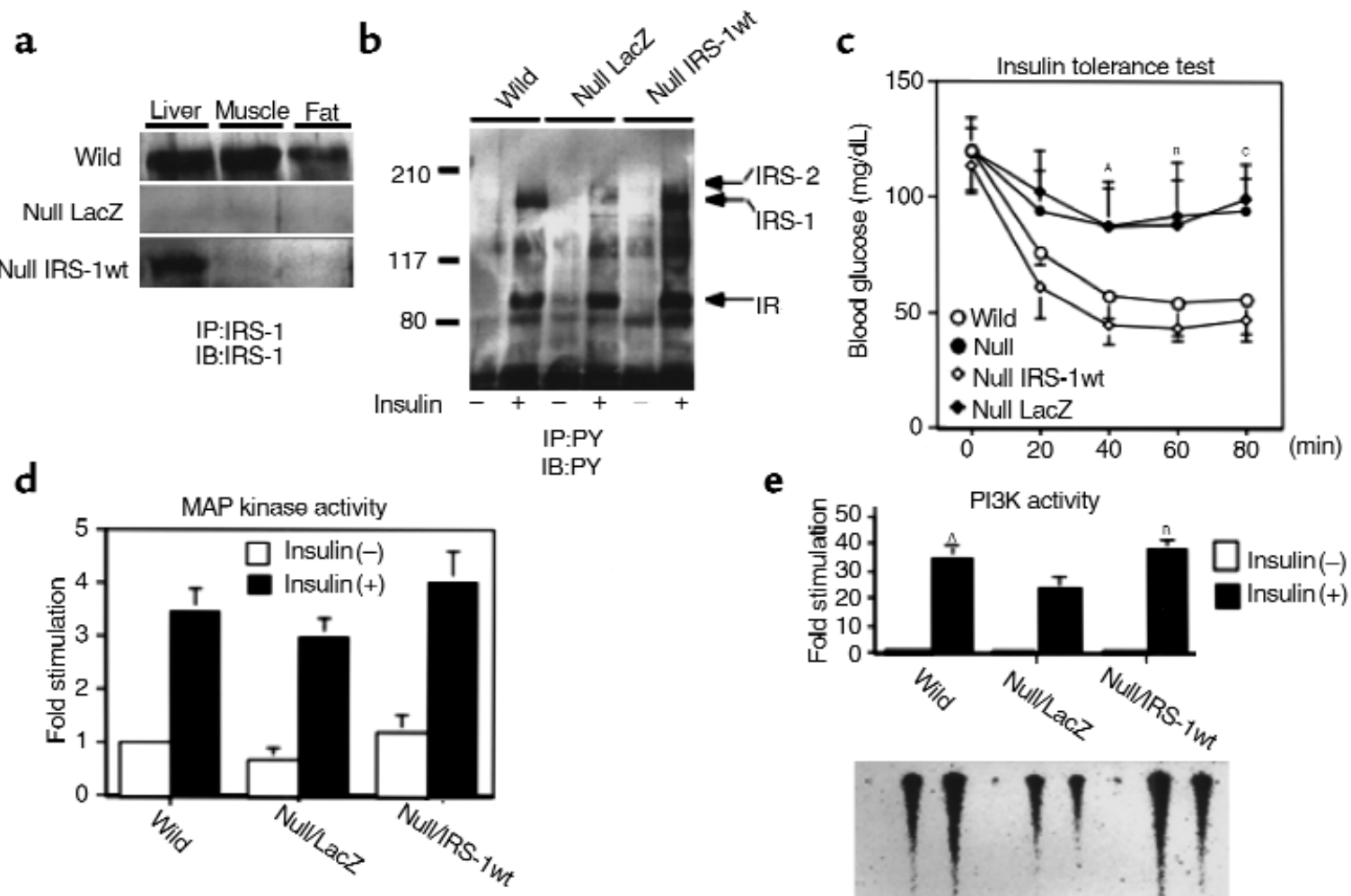

e

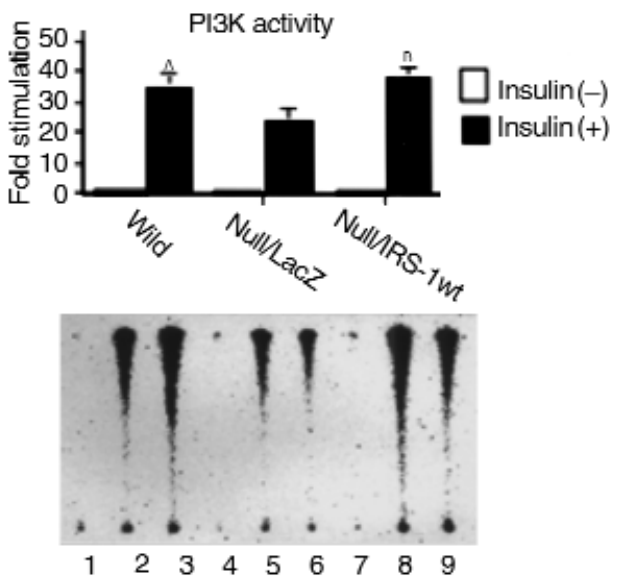

Figure 1

The effect of IRS-1 reconstitution by adenovirus-mediated gene transfer in the IRS-1-deficient mice. The IRS-1-deficient mice were treated with the adenoviruses as described in Methods. (a) Specific reconstitution of IRS-1 protein in the knockout mouse liver. The lysates of each tissue (liver, soleus muscle [muscle], and epididymal fat pad [fat]) from the indicated mouse (wild-type mouse [wild], null mouse treated with LacZ adenovirus [null LacZ], and null mouse treated with wild-type IRS-1 [null IRS-1wt]) were prepared as described in Methods. The lysates were immunoprecipitated (IP) with the antibodies against the COOH-terminal region of IRS-1 ( $\alpha$ IRS-1-CT) and subjected to SDS-PAGE (7\% gel), followed by Western blotting (IB) with the same antibodies. (b) Tyrosine-phosphorylated proteins in liver in response to insulin. Mice were anesthetized, and $1 \mu \mathrm{g} / \mathrm{g}$ body weight of insulin was injected through the inferior vena cava. After 5 minutes, the liver was removed and lysed with the tissue homogenization buffer as described in Methods. The lysates were immunoprecipitated with anti-phosphotyrosine antibodies (4G10) and subjected to SDS-PAGE (7\% gel), followed by Western blotting with the same antibodies. PY, anti-phosphotyrosine antibodies. (c) ITT on mice treated with the indicated adenoviruses. ITT was performed on the indicated group of mice (eight mice in each group). Each bar represents \pm SD. ${ }^{A} P<0.02$ wild-type or null IRS-1wt versus null or null LacZ. ${ }^{B} P<0.01$ wild-type or null IRS-1wt versus null or null LacZ. ${ }^{C} P<0.01$ wild-type or null IRS-1wt versus null or null LacZ. (d) Insulin-induced MAP kinase activity in the liver infected with the indicated adenoviruses. After being treated without or with insulin for 5 minutes, the liver lysates were immunoprecipitated with anti-MAP kinase antibodies ( $\alpha$ C92) and subjected to the immune complex kinase assay as described in Methods. The results are expressed as the ratio to the value of wild-type without insulin. Each bar represents the mean \pm SD of more than three independent experiments. (e) Insulin-induced PI3K activity associated with the tyrosine-phosphorylated proteins in the liver infected with the indicated adenoviruses. After being treated without or with insulin for 5 minutes, the liver lysates were immunoprecipitated with anti-phosphotyrosine antibodies and then subjected to PI3K assay as described in Methods. The lower panel shows the representative results (lanes 1-3; wild-type, lanes 4-6; null LacZ, lanes 7-9; null treated with IRS-1wt, lanes 1, 4, and 7; insulin [-], lanes 2, 3, 5, 6, 8, and 9; insulin [+]). In the upper panel, the results are expressed as the ratio to the value of wild-type without insulin, and each bar represents the mean \pm SD of more than three independent experiments. ${ }^{A} P<0.05$ wildtype insulin $(+)$ versus null LacZ insulin $(+) .{ }^{B} P<0.05$ null IRS- 1 wt insulin $(+)$ versus null LacZ insulin $\left(^{+}\right)$. 


\section{Figure 2}

Characterization of the IRS-1 mutant lacking p 85 PI3K binding sites. (a) Tyrosine phosphorylation of the wild-type (IRS-1wt) or the mutant IRS-1 (IRS-1 $\Delta$ p 85 ) in response to insulin. COS1 cells expressing human insulin receptors were infected with the indicated adenoviruses. After the infected cells were cultured in DMEM with $10 \%$ FCS for 48 hours, they were starved for 20 hours and treated with $100 \mathrm{nM}$ insulin for 5 minutes. The cell lysates were subjected to Western blot (IB) with anti-IRS antibodies (upper panel) or anti-phosphotyrosine antibodies (lower panel). (b) The ability of the wild-type or the mutant IRS-1 to interact with $\mathrm{SH} 2$ proteins. After insulin treatment, the cell lysates were subjected to pull-down study using GST-p85PI3K fusion protein (upper panel) or GST-Grb2 fusion protein (lower panel) as described in Methods, followed by Western blot with $\alpha$ IRS-1CT. (c) The effect of IRS-1wt or IRS- $1 \Delta \mathrm{p} 85$ expression on insulininduced PI3K activity in COS1 cells. After insulin treatment, the cell lysates were subjected to immunoprecipitation (IP) with antiphosphotyrosine antibodies followed by PI3K assay as described in Methods. The upper panel shows the representative result (lanes 1, 3, and 5; insulin [-], lanes 2, 4, and 6; insulin[+]). In the lower panel, the results are expressed as the ratio to the value of wild-type without insulin, and each bar represents the mean \pm SD of more than three independent experiments.

1-deficient mice. In the cells expressing LacZ, IRS-2 was the major tyrosine-phosphorylated protein stimulated by insulin (Figure 4, a and b). In the cells expressing IRS-1wt, IRS-1 was greatly tyrosine phosphorylated by insulin stimulation, and slight phosphorylation was seen even in the basal state, whereas phosphorylation of IRS-2 was abolished (Figure 4a). It is also likely that IRS-1 phosphorylation was relatively sustained; hence, IRS-2 phosphorylation was more transient (Figure $4 \mathrm{~b})$. Tyrosine phosphorylation of IRS- $1 \Delta \mathrm{p} 85$ is stronger than that of IRS- 2 in the cells expressing LacZ, but significantly decreased compared with that of IRS$1 \mathrm{wt}$, and phosphorylation of IRS-2 was undetectable (Figure 4a). Expression of IRS-1wt seemed to inhibit tyrosine phosphorylation of IRS-2 as well as did expression of IRS- $1 \Delta \mathrm{p} 85$ (Figure 4b). Furthermore, PI3K activity associated with tyrosine-phosphorylated proteins markedly increased in cells expressing IRS-1wt compared with that in cells expressing LacZ, whereas the activity in the cells expressing IRS- $1 \Delta \mathrm{p} 85$ seems to be as much as that in cells expressing LacZ (Figure 4c). Shc phosphorylation was not prominent in any cells by blotting with 4G10 using the cell lysates (Figure 4a).

Next, we investigated the effect of wild-type or the mutant IRS-1 expression on PKB activity that is thought to be a key enzyme regulating insulin's major metabolic responses downstream from $\operatorname{PI} 3 \mathrm{~K}(17,23$, 26). Expression of IRS-1wt in the hepatocytes from IRS-1-deficient mice increased insulin-induced PKB a

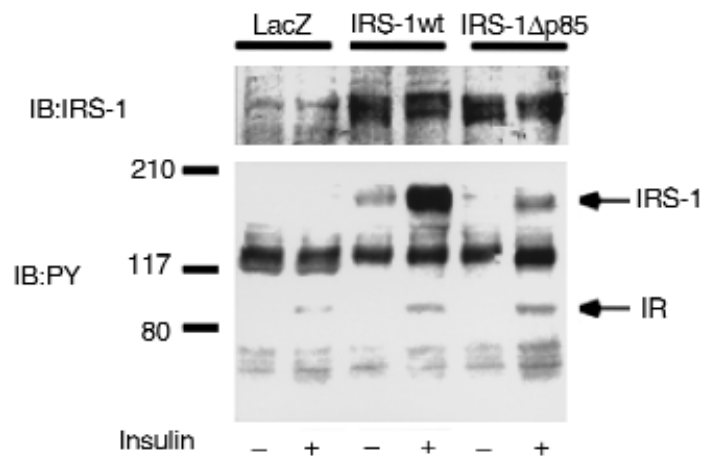

b

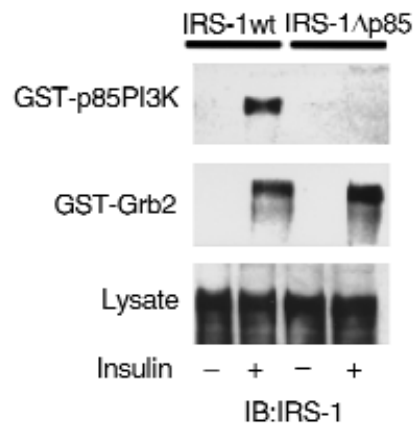

c

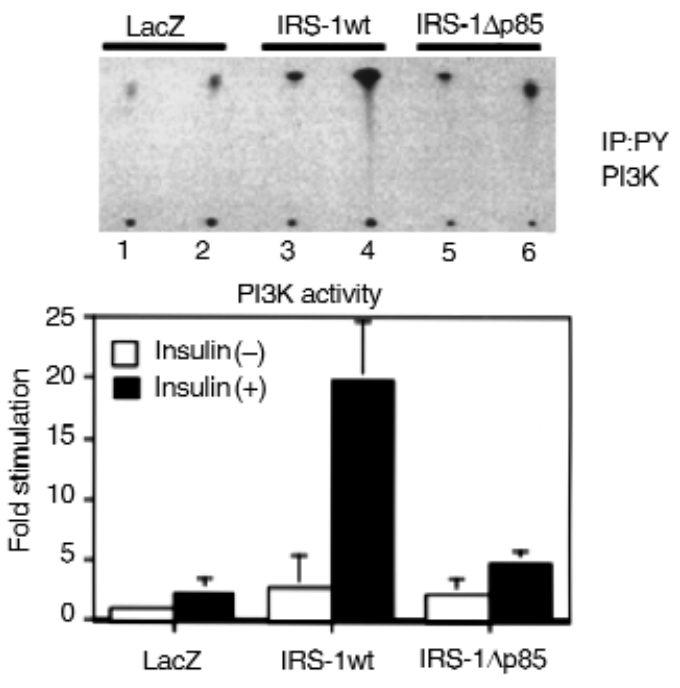

phosphorylation detected by mobility shift in Western blot, and its kinase activity determined by immune complex kinase assay, compared with the phosphorylation and the kinase activity in the cells expressing LacZ, respectively (Figure 5a). Interestingly, expression of IRS- $1 \Delta$ p 85 also promoted PKB activity to the almost same level as did IRS-1wt (Figure 5a). Furthermore, introduction of IRS- $1 w t$ or IRS- $1 \Delta \mathrm{p} 85$ into IRS-1-deficient mice also restored PKB activity in liver to the comparable level in the wild-type mice (Figure 5b). Taking into consideration an increase in PKB activity and the improvement of insulin sensitivity in the IRS1 -deficient mice expressing IRS- $1 w t$ or IRS- $1 \Delta \mathrm{p} 85$, 
Figure 3

The effect of the mutant of IRS-1 lacking PI3K binding sites on insulin sensitivity of IRS-1-deficient mice. (a) Introduction of IRS1 wt or IRS- $1 \Delta \mathrm{p} 85$ into the IRS-1-deficient mouse liver by adenovirus-mediated gene transfer. After insulin treatment, the liver was isolated and lysed as described. The lysates were immunoprecipitated (IP) with 4G10 (upper panel) or aIRS-1-CT (middle panel) and subjected to SDS-PAGE (7\% gel), followed by Western blotting (IB) with the same antibodies. The immunoprecipitates with 4G10 were also subjected to PI3K assay (lower panel). (b) ITT. ITT was performed against the indicated group of mice (eight mice in each group). Each bar represents \pm SD. ${ }^{A} P<0.02$ null IRS$1 \Delta \mathrm{p} 85$ versus null LacZ. ${ }^{\mathrm{B}} P<0.01$ IRS $-1 \Delta \mathrm{p} 85$ versus null LacZ.

enhancement of PKB might be essential for the recovery of insulin resistance in IRS-1-deficient mice. Recently, several reports have suggested that PKB is a candidate for the key regulator of gluconeogenesis or glycogen synthesis in liver $(27,28)$. However, it is unclear how PKB can be activated in liver even in the absence of full activation of PI3K. To explore the possible mechanism, we introduced IRS- 1 wt or IRS- $1 \Delta \mathrm{p} 85$ into the IRS-1-deficient hepatocytes with treatment of wortmannin because it is possible that even IRS- $1 \Delta \mathrm{p} 85$ could activate $\mathrm{PKB}$ via weak association with PI3K through other than YXXM motif or activation of PI3K by an unknown indirect mechanism independent of tyrosine phosphorylation. As Figure 5c shows, wortmannin strongly, but not completely, suppressed the effect of IRS-1wt on PKB activation, whereas it seemed less effective on PKB activation in cells expressing IRS$1 \Delta \mathrm{p} 85$. These data suggest that PI3K-dependent signal mostly contributes to activation of PKB under normal conditions and that wortmannin insensitive pathway might be partially involved in PKB activation in primary hepatocytes. They also suggest that IRS- $1 \Delta \mathrm{p} 85$ can fully activate PKB without full activation of PI3K.

\section{Discussion}

We previously generated IRS-1-deficient mice and revealed that the disruption of IRS-1 results in the peripheral insulin resistance owing to the disturbance of the signaling cascades from IRS-1 $(3,29,30)$. Howev$\mathrm{er}$, it is still unclear which tissue mainly contributes to developing the whole-body insulin resistance, one of the important risk factors of diabetes. In this study, we try to elucidate the importance of liver on the whole-body insulin resistance due to disruption of IRS- 1 and also challenge the liver-specific gene therapy to the IRS1-deficient mice. To this end, we express the wild-type and a mutant IRS- 1 into these animals by adenovirus- a

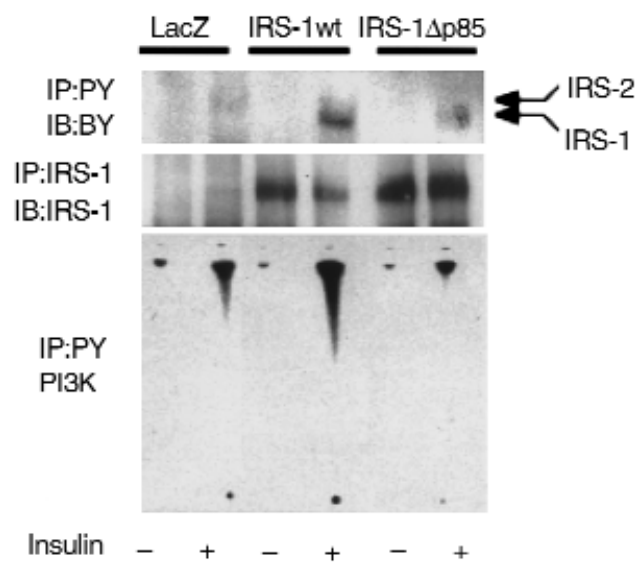

b

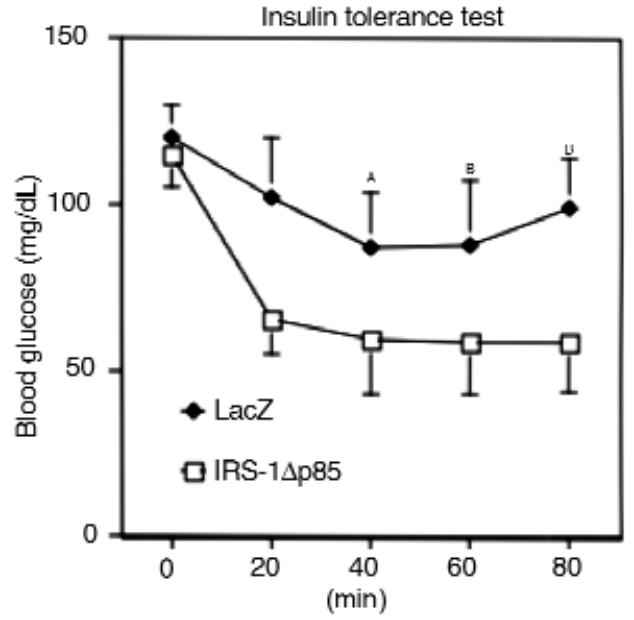

mediated gene transfer. The introduced IRS- 1 is mainly detected in liver as expected. Interestingly, restoration of IRS- 1 only in liver results in almost complete recovery from the whole-body insulin resistance, whereas disturbance of insulin signaling in the skeletal muscle of IRS-1-deficient mice seems to be more severe than that in the liver (29). Recently, Bruning et al. have demonstrated that skeletal muscle-specific insulin receptor knockout does not affect the whole-body glucose homeostasis, whereas the knockout mice show severe insulin resistance in their skeletal muscle (11). Taken together, our data suggest that IRS proteins play a pivotal role in glucose homeostasis presumably through regulating insulin-induced inhibition of gluconeogenesis or promotion of glycogen synthesis or both.

What is the most important signal from IRS-1 in liver to maintain glucose homeostasis? Comparing insulininduced PI3K and MAPK in IRS-1-deficient mice, the major IRS protein-mediated signaling events, the former is much more decreased as shown previously (3). In addition, PI3K has been shown to play a pivotal role in a variety of insulin's metabolic actions (23). Thus, we express an IRS-1 mutant lacking p85 subunit of PI3K, IRS- $1 \Delta \mathrm{p} 85$, in IRS-1-deficient mice in order to explore the role of PI3K in liver on systemic glucose homeosta- 


\section{Figure 4}

Characterization of IRS-1wt- or IRS-1 $\Delta \mathrm{p} 85$-mediated signaling events in IRS-1-deficient primary hepatocytes. (a) Tyrosine-phosphorylated proteins in the IRS-1-deficient primary hepatocytes expressing the indicated constructs. Cells were infected with adenovirus (moi 10) and cultured for 24 hours without serum as described in Methods. After being treated without or with $100 \mathrm{nM}$ insulin for 1 minute, cell lysates were subjected to SDS-PAGE, followed by Western blot analysis (IB) with 4G10 (7\% gel). (b) Time course of tyrosine phosphorylation on IRS proteins in the IRS1-deficient primary hepatocytes expressing the indicated constructs. After being treated without or with $100 \mathrm{nM}$ insulin for the indicated periods, cell lysates were immunoprecipitated (IP) with 4G10 (upper panel) or $\alpha$ IRS-1-CT (lower panel), and then the immunoprecipitates were subjected to SDS-PAGE, followed by Western blot analysis with the same antibodies. (c) Insulin-induced PI3K activity in the IRS-1-deficient primary hepatocytes expressing the indicated constructs. After being treated without or with insulin for the indicated periods, the cell lysates were immunoprecipitated with 4G10 and subjected to PI3K assay. The upper panel shows the representative result. In the lower panel, the results are expressed as the ratio to the value of LacZ without insulin, and each bar represents the mean \pm SD of more than three independent experiments. ${ }^{A} P<0.01$ LacZ insulin (+) versus IRS- 1 wt insulin $(+) .{ }^{B} P<0.01$ IRS- $1 \Delta$ p 85 insulin $(+)$ versus IRS-1wt insulin (+).

sis. Contrary to the expectation, expression of IRS$1 \Delta \mathrm{p} 85$ normalizes insulin sensitivity of IRS-1-deficient mice as well as that of IRS-1wt. Expression of IRS$1 \Delta \mathrm{p} 85$ does not increase the PI3K activity associated with tyrosine-phosphorylated proteins in either the IRS-1-deficient mouse liver or the primary hepatocytes, although we cannot exclude the possibility that IRS- $1 \Delta$ p 85 promotes PI $3 \mathrm{~K}$ activity independent of tyrosine phosphorylation by an unknown mechanism(s). However, IRS- $1 \Delta \mathrm{p} 85$ promotes PKB activity as much as does IRS-1wt, although PKB has been shown to lie downstream of PI3K in various types of cells (26). The activation mechanism of PKB mediated by IRS$1 \Delta \mathrm{p} 85$ is unclear. Full activation of $\mathrm{PI} 3 \mathrm{~K}$ is not required for activation of PKB at least in cells expressing IRS$1 \Delta \mathrm{p} 85$, whereas PI3K mostly contributes to activation of PKB in cells expressing IRS-1wt (Figure 5c). However, it is also possible that the very small amount of PI3K activity associated with IRS proteins is sufficient for the full activation of PKB because of the particular subcellular localization of IRS/PI3K complex. Nevertheless, it is likely that in the liver and the primary hepatocytes, IRS- 1 mediates the signals to activation of PKB by the PI3K-dependent pathway and the PI3K-independent pathway(s), such as the Ras-dependent one $(31,32)$. Recently, Uchida et al. have demonstrated that a

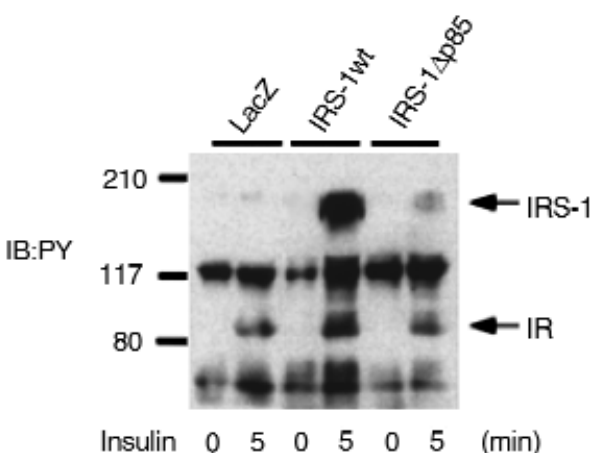

b

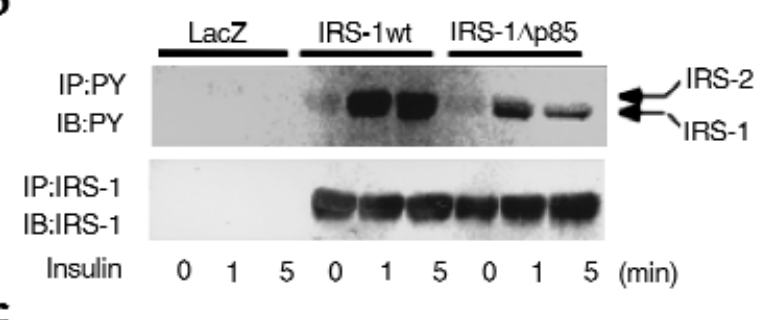

c
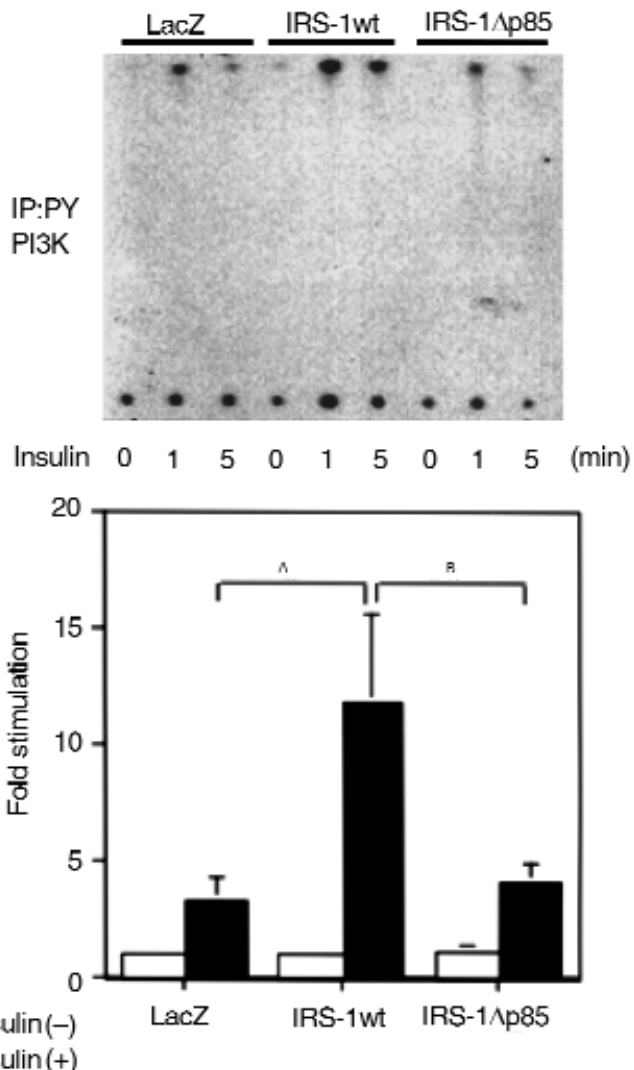

IRS-4, a member of insulin receptor substrates, promotes full activation of $\mathrm{PKB}$, although it associates with a small amount of PI3K activity (33), suggesting that IRS protein could transmit the signal to activation of PKB without full activation of PI3K. In any case, our data suggest the possibility that full activation of PKB in liver is sufficient for systemic glucose homeostasis without full activation of PI3K.

Hepatic glucose production is known to play a cru- 
Figure 5

PKB activity in IRS-1-deficient primary hepatocytes and liver. (a) The effect of IRS-1 wt or IRS$1 \Delta \mathrm{p} 85$ on PKB phosphorylation and activity in IRS-1-deficient primary hepatocytes. After being treated without or with $100 \mathrm{nM}$ insulin for 5 minutes, the cell lysates were subjected to SDS-PAGE, followed by Western blot analysis (IB) with anti-PKB antibodies ( $\alpha$ PKB-CT) (upper panel), or subjected to immunoprecipitation (IP) with $\alpha$ PKB-CT followed by the immune complex kinase assay as described in Methods (lower panel). The results are expressed as the ratio to the value of Lac $Z$ without insulin, and each bar represents the mean \pm $\mathrm{SD}$ of more than three independent experiments. ${ }^{A} P<0.05 \mathrm{LacZ}$ insulin (+) versus IRS- $1 \mathrm{wt}$ insulin $(+) .{ }^{B} P<0.05$ LacZ insulin (+) versus IRS$1 \Delta \mathrm{p} 85$ insulin $(+)$. (b) The effect of IRS-1wt or IRS-1 1 p 85 on PKB activity in IRS-1-deficient mouse liver. After being treated without or with insulin for 5 minutes, the liver lysates were immunoprecipitated with $\alpha$ PKB-CT and then subjected to PKB kinase assay. The results are expressed as the ratio to the value of Lac $Z$ without insulin, and each bar represents the mean \pm SD of more than three independent experiments. ${ }^{A} P<0.01 \mathrm{Lac} Z$ insulin (+) versus wildtype insulin $(+) .{ }^{B} P<0.05$ LacZ insulin $(+)$ versus IRS- 1 wt insulin $(+) .{ }^{C} P<0.05 \mathrm{LacZ}$ insulin $(+)$ versus IRS- $1 \Delta$ p 85 insulin $(+)$. (c) The effect of PI3K inhibition on PKB activation on IRS-1 deficient primary hepatocytes. The indicated proteins were introduced into IRS-1-deficient primary hepatocytes by adenovirus-mediated gene transfer. Cells were incubated with or without $50 \mathrm{nM}$ wortmannin for 30 minutes and were treated with $100 \mathrm{nM}$ insulin for 5 minutes. The cell lysates were subjected to immunoprecipitation with 4G10 or $\alpha$ PKB-CT, followed by PI3K assay (upper panel) and PKB immune complex kinase assay (lower panel), respectively. In the lower panel, the results are expressed as the ratio to the value of LacZ without insulin, and each bar represents the mean of two experiments. wor, wortmannin.

cial role for the systemic glucose homeostasis. Insulin decreases this by activating glycogen synthesis and glycolysis and by inhibiting gluconeogenesis. Glycogen synthesis in liver is mainly regulated by glucose input that correlates with blood glucose level. However, insulin also participates in the glycogen synthesis regulation presumably through the PI3K-dependent pathway (34), although it remains unclear which molecules are involved in this glycogen synthesis regulation. In skeletal muscle, PI3K/PKB/GSK3 cascade seems to be one of the key players in the regulation of glycogen synthase activity $(17,35)$. Thus, it is possible that this cascade is also involved in the regulation of glycogen synthase activity in liver. Indeed, PKB has been recently reported to be a candidate for the kinase regulating glycogen synthesis in rat primary hepatocytes (28). In glycolysis steps, PI3K seems to activate 6-phosphofructose 2-kinase (PFK2) through phosphorylation in response to insulin, leading to generation of fructose 2,6-bisphosphate, a key allosteric activator of PFK1, the rate-limiting factor of glycolysis (36). Recently PKB has been shown to directly phosphorylate and activate PFK2 in vitro (37). Therefore, PKB might be an important regulator of insulin-dependent glycolysis. In gluconeogenesis in liver, phosphoenolpyruvate carboxykinase (PEPCK) expression is the rate-limiting step, primarily regulated at the transcription level. Insulin inhibits glucagon and glucocorticoid-induced PEPCK expression presumably through PI3K activity $(38,39)$. Some reports suggested that PKB is involved in this regulation, whereas others showed the opposite results $(27,40)$. In any case, it is possible that PKB could contribute to reduce the hepatic glucose production by at least one of these mechanisms.

In conclusion, our results presented here show that restoration of IRS- 1 in liver is sufficient for normalization of systemic insulin resistance in IRS-1-deficient mice, suggesting that regulating hepatic glucose production is pivotal for systemic glucose homeostasis. Furthermore, they suggest that PKB activity mediated by IRS- 1 might play a crucial role in this regulation. Additionally, our results have also demonstrated that 
adenovirus-mediated gene therapy is effective to normalize systemic insulin resistance at least under the particular conditions, presenting the possibilities of new therapy for the some types of diabetes.

\section{Acknowledgments}

We thank C.R. Kahn (Joslin Diabetes Center) for helpful comments, I. Saito for adenovirus vector, and S. Kakinuma for technical assistance. This work was supported by Grant-in-aid for Creative Basic Research 10NP0201 from the Ministry of Education, Science, Sports, and Culture, Japan (to T. Kadowaki).

1. Kahn, C.R. 1994. Banting Lecture. Insulin action, diabetogenes, and the cause of type II diabetes. Diabetes. 43:1066-1084.

2. Kahn, C.R. 1995. Diabetes. Causes of insulin resistance. Nature. 373:384-385.

3. Tamemoto, H., et al. 1994. Insulin resistance and growth retardation in mice lacking insulin receptor substrate-1. Nature. 372:182-186.

4. Araki, E., et al. 1994. Alternative pathway of insulin signalling in mice with targeted disruption of the IRS-1 gene. Nature. 372:186-190.

5. Terauchi, Y., et al. 1997. Development of non-insulin-dependent diabetes mellitus in the double knockout mice with disruption of insulin receptor substrate- 1 and beta cell glucokinase genes. Genetic reconstitution of diabetes as a polygenic disease. J. Clin. Invest. 99:861-866.

6. Bruning, J.C., et al. 1997. Development of a novel polygenic model of NIDDM in mice heterozygous for IR and IRS-1 null alleles. Cell. 88:561-572.

7. Withers, D.J., et al. 1998. Disruption of IRS-2 causes type 2 diabetes in mice. Nature. 391:900-904.

8. Kahn, B.B. 1998. Type 2 diabetes: when insulin secretion fails to compensate for insulin resistance. Cell. 92:593-596.

9. Haring, H.U., and Mehnert, H. 1993. Pathogenesis of type 2 (non-insulindependent) diabetes mellitus: candidates for a signal transmitter defect causing insulin resistance of the skeletal muscle. Diabetologia. 36:176-182.

10. Beck-Nielsen, H., Hother-Nielsen, O., Vaag, A., and Alford, F. 1994. Pathogenesis of type 2 (non-insulin-dependent) diabetes mellitus: the role of skeletal muscle glucose uptake and hepatic glucose production in the development of hyperglycaemia. A critical comment. Diabetologia. 37:217-221.

11. Bruning, J.C., et al. 1998. A muscle-specific insulin receptor knockout exhibits features of the metabolic syndrome of NIDDM without altering glucose tolerance. Mol. Cell. 2:559-569.

12. Moller, D.E., et al. 1996. Transgenic mice with muscle-specific insulin resistance develop increased adiposity, impaired glucose tolerance, and dyslipidemia. Endocrinology. 137:2397-2405.

13. Lauro, D., et al. 1998. Impaired glucose tolerance in mice with a targeted impairment of insulin action in muscle and adipose tissue. Nat. Genet. 20:294-298.

14. Peeters, M.J., Patijn, G.A., Lieber, A., Meuse, L., and Kay, M.A. 1996. Adenovirus-mediated hepatic gene transfer in mice: comparison of intravascular and biliary administration. Hum. Gene Ther. 7:1693-1699.

15. Herz, J., and Gerard, R.D. 1993. Adenovirus-mediated transfer of low density lipoprotein receptor gene acutely accelerates cholesterol clearance in normal mice. Proc. Natl. Acad. Sci. USA. 90:2812-2816.

16. Yamamoto-Honda, R., et al. 1996. Mutant of insulin receptor substrate1 incapable of activating phosphatidylinositol 3-kinase did not mediate insulin-stimulated maturation of Xenopus laevis oocytes. J. Biol. Chem. 271:28677-28681.

17. Ueki, K., et al. 1998. Potential role of protein kinase B in insulin-induced glucose transport, glycogen synthesis, and protein synthesis. J. Biol. Chem. 273:5315-5322.

18. Ledley, F.D., Darlington, G.J., Hahn, T., and Woo, S.L. 1987. Retroviral gene transfer into primary hepatocytes: implications for genetic therapy of liver-specific functions. Proc. Natl. Acad. Sci. USA. 84:5335-5339.

19. Ueki, K., et al. 1994. Feedback regulation of mitogen-activated protein kinase kinase kinase activity of c-Raf-1 by insulin and phorbol ester stimulation. J. Biol. Chem. 269:15756-15761.

20. Tobe, K., et al. 1995. Identification of a $190-\mathrm{kDa}$ protein as a novel substrate for the insulin receptor kinase functionally similar to insulin receptor substrate-1. J. Biol. Chem. 270:5698-5701.

21. Miyake, S., et al. 1996. Efficient generation of recombinant adenoviruses using adenovirus DNA-terminal protein complex and a cosmid bearing the full-length virus genome. Proc. Natl. Acad. Sci. USA. 93:1320-1324.

22. Abe, H., et al. 1998. Hypertension, hypertriglyceridemia, and impaired endothelium-dependent vascular relaxation in mice lacking insulin receptor substrate-1. J. Clin. Invest. 101:1784-1788.

23. Shepherd, P.R., Withers, D.J., and Siddle, K. 1998. Phosphoinositide 3kinase: the key switch mechanism in insulin signalling. Biochem. J. 333:471-490.

24. Terauchi, Y., et al. 1999. Increased insulin sensitivity and hypoglycaemia in mice lacking the 85 alpha subunit of phosphoinositide 3-kinase. Nat. Genet. 21:230-235.

25. Sun, X.J., et al. 1991. Structure of the insulin receptor substrate IRS-1 defines a unique signal transduction protein. Nature. 352:73-77.

26. Coffer, P.J., Jin, J., and Woodgett, J.R. 1998. Protein kinase B (c-Akt): a multifunctional mediator of phosphatidylinositol 3-kinase activation. Biochem. J. 335:1-13.

27. Liao, J., Barthel, A., Nakatani, K., and Roth, R.A. 1998. Activation of protein kinase B/Akt is sufficient to repress the glucocorticoid and cAMP induction of phosphoenolpyruvate carboxykinase gene. J. Biol. Chem. 273:27320-27324.

28. Peak, M., Rochford, J.J., Borthwick, A.C., Yeaman, S.J., and Agius, L. 1998. Signalling pathways involved in the stimulation of glycogen synthesis by insulin in rat hepatocytes. Diabetologia. 41:16-25.

29. Yamauchi, T., et al. 1996. Insulin signalling and insulin actions in the muscles and livers of insulin-resistant, insulin receptor substrate 1-deficient mice. Mol. Cell. Biol. 16:3074-3084.

30. Kaburagi, Y., et al. 1997. Role of insulin receptor substrate-1 and pp60 in the regulation of insulin-induced glucose transport and GLUT4 translocation in primary adipocytes. J. Biol. Chem. 272:25839-25844.

31. Franke, T.F., et al. 1995. The protein kinase encoded by the Akt protooncogene is a target of the PDGF-activated phosphatidylinositol 3kinase. Cell. 81:727-736.

32. van Weering, D.H., et al. 1998. Protein kinase B activation and lamellipodium formation are independent phosphoinositide 3-kinase-mediated events differentially regulated by endogenous Ras. Mol. Cell. Biol. 18:1802-1811.

33. Uchida, T., Myers, M.G., Jr., and White, M.F. 2000. IRS-4 mediates protein kinase B signaling during insulin stimulation without promoting antiapoptosis. Mol. Cell. Biol. 20:126-138.

34. Bollen, M., Keppens, S., and Stalmans, W. 1998. Specific features of glycogen metabolism in the liver. Biochem. J. 336:19-31.

35. Cross, D.A., Alessi, D.R., Cohen, P., Andjelkovich, M., and Hemmings, B.A. 1995. Inhibition of glycogen synthase kinase- 3 by insulin mediated by protein kinase B. Nature. 378:785-789.

36. Lefebvre, V., Mechin, M.C., Louckx, M.P., Rider, M.H., and Hue, L. 1996. Signaling pathway involved in the activation of heart 6-phosphofructo2- kinase by insulin. J. Biol. Chem. 271:22289-22292.

37. Deprez, J., Vertommen, D., Alessi, D.R., Hue, L., and Rider, M.H. 1997. Phosphorylation and activation of heart 6-phosphofructo-2-kinase by protein kinase $B$ and other protein kinases of the insulin signaling cascades. J. Biol. Chem. 272:17269-17275.

38. Sutherland, C., O'Brien, R.M., and Granner, D.K. 1995. Phosphatidylinositol 3-kinase, but not $\mathrm{p} 70 / \mathrm{p} 85$ ribosomal S6 protein kinase, is required for the regulation of phosphoenolpyruvate carboxykinase (PEPCK) gene expression by insulin. Dissociation of signaling pathways for insulin and phorbol ester regulation of PEPCK gene expression. J. Biol. Chem. 270:15501-15506

39. Gabbay, R.A., et al. 1996. Insulin regulation of phosphoenolpyruvate carboxykinase gene expression does not require activation of the Ras/mitogen-activated protein kinase signaling pathway. J. Biol. Chem. 271:1890-1897.

40. Agati, J.M., Yeagley, D., and Quinn, P.G. 1998. Assessment of the roles of mitogen-activated protein kinase, $\mathrm{PI} 3 \mathrm{~K}$, protein kinase $\mathrm{B}$, and protein kinase $\mathrm{C}$ in insulin inhibition of CAMP-induced phosphoenolpyruvate carboxykinase gene transcription. J. Biol. Chem. 273:18751-18759. 